\title{
Pulses and Fronts in the Complex Ginzburg-Landau Equation near a Subcritical Bifurcation
}

\author{
W. van Saarloos and P. C. Hohenberg \\ AT\&T Bell Laboratories, Murray Hill, New Jersey 07974
}

(Received 30 October 1989)

\begin{abstract}
Uniformly translating solutions of the one-dimensional complex Ginzburg-Landau equation are studied near a subcritical bifurcation. Two classes of solutions are singled out since they are often produced starting from localized initial conditions: moving fronts and stationary pulses. A particular exact analytic front solution is found, which is conjectured to control the relative stability of pulses and fronts. Numerical solutions of the Ginzburg-Landau equation confirm the predictions based on this conjecture.
\end{abstract}

PACS numbers: $47.10 .+\mathrm{g}, 05.45 .+\mathrm{b}, 47.20 . \mathrm{Ky}, 47.25 . \mathrm{Qv}$

Spatially extended nonequilibrium systems often show coherent structures formed from the spatial juxtaposition of different types of solutions, particularly near subcritical bifurcations where the different solutions are individually stable. Examples are moving fronts formed when a stable state invades an unstable one and, in the bistable case, pulses formed by bubbles of one state embedded in the other, or fronts between stable states. When the dynamics of the system is characterized by a minimizing potential (Lyapunov function) the behavior of such structures can often be inferred by comparing the values of the potential for each of the states, but in the opposite case when no Lyapunov functional exists the situation is much more complicated and there exists a rich variety of different structures with often surprising behavior. For example, pulses and fronts appear in binary-fluid convection,' ${ }^{1}$ plane Poiseuille flow, ${ }^{2,3}$ and Taylor-Couette flow with counter-rotating cylinders, ${ }^{4}$ as well as in numerical simulations of model systems. ${ }^{3,5}$ Depending on parameter values these coherent structures are found to vary either periodically or chaotically in time, and to have spatial envelopes which may be stationary or uniformly moving, or may undergo chaotic motion. The present work summarizes results of a comprehensive analytic and numerical study ${ }^{6}$ of a simple equation displaying all of the above types of behavior: the one-dimensional complex ${ }^{7}$ Ginzburg-Landau (GL) equation near a subcritical bifurcation.

In general, it is found that for given parameter values a multiplicity of front and pulse solutions exists. Our aim is to understand this multiplicity and especially to elucidate the ensuing selection problem: Which solution will be reached starting from specified initial conditions? For the real equation, ${ }^{7}$ as well as for other cases with a Lyapunov function, this selection problem only arises above the bifurcation point $(\epsilon>0)$, where earlier work ${ }^{8}$ has shown that the form of the selected front is correctly predicted by simple criteria which go by the name "linear and nonlinear marginal stability" [see Figs. 1 (a) and $1(\mathrm{~b})$ ]. Our objective is to generalize these criteria to the complex case and to the bistable regime, $\epsilon<0$. In addition to moving fronts, the complex equation has been shown numerically to possess both periodic ${ }^{5}$ and chaotic ${ }^{3}$ pulse solutions for certain parameter values in the bistable regime. Our main result is the discovery of an exact "selected" front solution which allows us to predict analytically whether a pulse or a front will be preferred, and in the latter case what the front velocity will be.

The one-dimensional complex GL equation may, by a suitable choice of units, be written in the general form

$$
\begin{aligned}
\partial_{t} A=\epsilon & A+\left(1+i c_{1}\right) \partial_{x}^{2} A \\
& +\left(1+i c_{3}\right)|A|^{2} A-\left(1-i c_{5}\right)|A|^{4} A,
\end{aligned}
$$
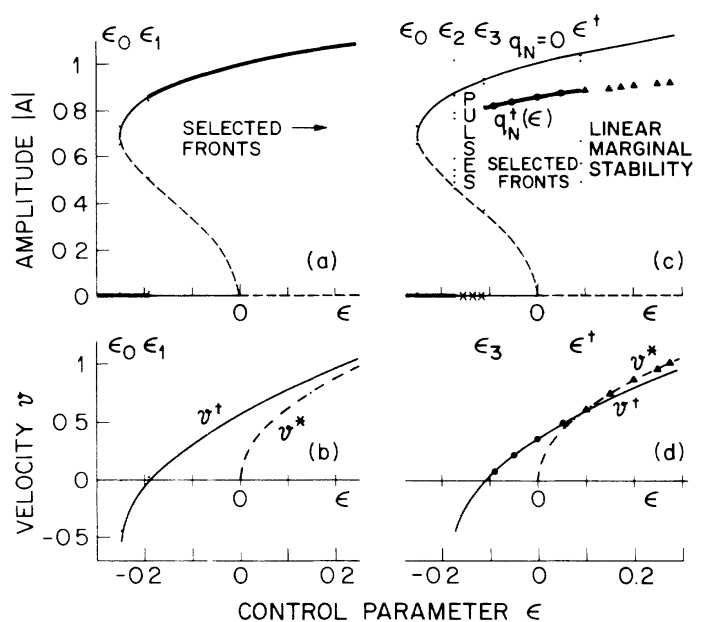

FIG. 1. (a),(c) Bifurcation diagrams and (b),(d) front velocity as a function of control parameter. (a) and (b) refer to the real GL equation $\left[c_{1}=0\right.$ in (1)], which is bistable in the range $\epsilon_{0}<\epsilon<0$. Dashed lines refer to unstable solutions, solid lines to stable ones. For $\epsilon>\epsilon_{1}=-\frac{3}{16}$ the selected front [thick line in (a)] has velocity $v^{+}>0$ which is larger than the velocity $v^{*}$ given by linear marginal stability [dot-dashed line in (b)]. For $\epsilon=\epsilon^{\dagger}=\frac{3}{4}$ (not shown) $v^{*}=v^{+}$and for $\epsilon>\epsilon^{\dagger}$ the linear front is selected. For $\epsilon<\epsilon_{1}, v^{+}<0$, the $|A|=0$ state invades the $|A| \neq 0$ one [thick line in (a)]. (c) and (d) refer to the complex case $\left[c_{1}=-0.1, c_{3}=0.2, c_{5}=0.15\right.$ in (1)], for which the front with velocity $v^{+}$and wave vector $q_{N}^{+}$is selected in the range $\epsilon_{3}<\epsilon<\epsilon^{\dagger}$. Solid circles are results of numerical solution of (1). For $\epsilon>\epsilon^{\dagger}, v^{*}>v^{+}$, and we have linear marginal stability (solid triangles), whereas for $\epsilon<\epsilon_{3}, v^{\dagger}<0$ and stable pulses, denoted by crosses, are found for $\epsilon_{2}<\epsilon<\epsilon_{3}$. For $\epsilon<\epsilon_{2}$ the $|A|=0$ state invades all $A \neq 0$ states. 
where $A(x, t)$ is complex, $\epsilon, c_{1}, c_{3}, c_{5}$ are real parameters, and we have chosen a frame of reference where there is no $\partial_{x} A$ term and no imaginary linear term $i c_{0} A$. An important class of solutions are the uniformly translating profiles

$$
A(x, t)=e^{-i \omega t} \hat{A}(x-v t)=e^{-i \omega t} a(\xi) e^{i \phi(\xi)},
$$

where $\xi=x-v t$. Upon introducing the quantities

$$
q=\partial_{\xi} \phi, \kappa=a^{-1} \partial_{\xi} a,
$$

Eq. (1) becomes $\left(a^{\prime}=\partial_{\xi} a\right.$, etc. $)$

$$
a^{\prime}=\kappa a, \quad \kappa^{\prime}=K, \quad q^{\prime}=Q,
$$

with

$$
\begin{aligned}
K= & -\tilde{\epsilon}-c_{1} \tilde{\omega}-\tilde{v} \kappa-c_{1} \tilde{v} q+q^{2}-\kappa^{2} \\
& -\left(1+c_{1}^{2}\right)^{-1}\left[\left(1+c_{1} c_{3}\right) a^{2}-\left(1-c_{1} c_{5}\right) a^{4}\right] \\
Q= & c_{1} \tilde{\epsilon}-\tilde{\omega}+c_{1} \tilde{v} \kappa-\tilde{v} q-2 \kappa q \\
& +\left(\tilde{c}_{1}-\tilde{c}_{3}\right) a^{2}-\left(\tilde{c}_{1}+\tilde{c}_{5}\right) a^{4}
\end{aligned}
$$

and $\tilde{\alpha} \equiv \alpha\left(1+c_{1}^{2}\right)^{-1}$ for any parameter $\alpha$. The threevariable dynamical system (4) has fixed points corresponding to uniformly translating solutions of the GL equation (1) which are periodic in space and time. There are two classes of such fixed points, the finiteamplitude "nonlinear" ones $(N)$ with $a_{N} \neq 0, q_{N} \neq 0$, and $\kappa_{N}=0$, and "linear" ones $(L)$ with $a_{L}=0, q_{L} \neq 0$, and $\kappa_{L} \neq 0$. The dependence of $a_{N}$ on $\epsilon$ for the nonlinear solution with $q_{N}=0$ is shown in Fig. 1(a) for the real equation and in Fig. 1 (c) for the complex one. In both cases $^{7}$ there exists in addition a band of solutions $a_{N}\left(q_{N}, \epsilon\right)=a_{N}\left(0, \epsilon-q_{N}^{2}\right)$ for any $q_{N}$, but the $q_{N} \neq 0$ states turn out to be dynamically relevant only for the complex equation. Expressions for $a_{N}, q_{N}, q_{L}, \kappa_{L}$ are readily obtained by solving the fixed-point equations $a^{\prime}=\kappa^{\prime}=q^{\prime}=0$. Besides the fixed points $N$ and $L$ there exist so-called "coherent structures" which are uniformly translating solutions of (1) with spatially varying envelopes. These correspond to (heteroclinic) trajectories ${ }^{2}$ of (4) joining different fixed points. Let us denote by $L_{ \pm}$the linear fixed-point solutions with $\kappa_{L} \gtrless 0$, respectively; for increasing $\xi$ trajectories near these points correspond to growth $\left(L_{+}\right)$or decay $\left(L_{-}\right)$of the amplitude $a$ away from zero. Then we can distinguish three types of coherent structures: ${ }^{9}$ pulses going from $L_{+}$to $L_{-}$; fronts going from $N$ to $L-$ (or $L+$ to $N$ ); and domain walls which join different $N$ fixed points (we shall not discuss domain walls further here).

As is well known, ${ }^{2}$ the condition for existence of a heteroclinic trajectory is that the stable and unstable manifolds of the fixed points in question should join up. It is thus possible to determine the multiplicity of the aforementioned coherent structures by studying the linear stability of the fixed points in the dynamics of Eq. (4). It should be emphasized, of course, that such argu- ments do not prove the existence or nonexistence of solutions, only their multiplicity, i.e., the likelihood of finding a nearby solution if one is known to exist. The stability analysis of the fixed points of (4) leads to the following predictions: ${ }^{2}$ For fixed values ${ }^{10}$ of $\epsilon$ and the coefficients $c_{i}$, there is a family of fronts with frequencies $\omega_{f}(v)$ and a continuum of velocities; this family is associated with one of the nonlinear fixed points $\left(N_{1}\right.$, say). From this family we expect to find stationary fronts $\omega_{\mathrm{sf}}=\omega_{f}(v=0)$ at generic points in parameter space. In addition, there is a discrete set of fronts with specified velocity $v^{\dagger}$ and frequency $\omega^{\dagger}$, associated with the other nonlinear fixed point $N_{2}$. One member of this discrete set will turn out to be the "selected front" mentioned above. For $\epsilon<0$ and fixed $c_{i}$, one also finds a discrete set of moving pulse solutions with specified velocity $v_{p}$ and frequency $\omega_{p}$, plus a symmetric stationary pulse $\left(v_{\mathrm{sp}}=0, \omega_{\mathrm{sp}}\right)$ which exists for generic parameter values.

We have constructed particular solutions of (4) representing exact fronts and pulses. The front solution is analogous to the one found earlier ${ }^{8}$ for the real equation. Its form is ${ }^{11}$

$$
\begin{aligned}
& \kappa=\kappa_{L}\left(1-a^{2} / a_{N}^{2}\right), \\
& q=q_{L}+\left(q_{N}-q_{L}\right) a^{2} / a_{N}^{2},
\end{aligned}
$$

the six constants $\omega, v, \kappa_{L}, q_{L}, q_{N}, a_{N}$ being determined ${ }^{6}$ by inserting (5) into (4). It can be shown ${ }^{6}$ that this solution belongs to the discrete set $v^{\dagger}, \omega^{\dagger}$, rather than to the family $\omega_{f}(v)$. The stationary pulse is a generalization of the solution of Hocking and Stewartson ${ }^{2,12}$ which here takes the form

$$
\begin{aligned}
& \kappa^{2}=\kappa_{L}^{2}\left(1-a^{2} / a_{0}^{2}\right)\left(1+d_{0} a^{2} / a_{0}^{2}\right), \\
& q=d_{1} \kappa .
\end{aligned}
$$

When (6) is inserted into (4) one finds ${ }^{6}$ six equations for the five real constants $\kappa_{L}, a_{0}>0, d_{0}>-1, d_{1}$, and $\omega$, so that the Ansatz (6) is only valid in a codimension-one subspace of the parameter space $\left(c_{1}, c_{3}, c_{5}\right)$, though as mentioned above, we expect stationary pulses to exist throughout the parameter space. From (5) and (6) it is easy to find analytic expressions ${ }^{6}$ for the pulse and front shapes $a(\xi), q(\xi)$. In contrast to the front solution obtained by the Ansatz (5), it turns out that the pulse solution given by $(6)$ is never stable.

The most interesting question about fronts and pulses is their dynamical behavior as solutions of Eq. (1), namely their stability and the ease with which they can be reached from given initial conditions (their basin of attraction). Although we cannot claim to have fully solved this difficult selection problem, we have generalized the rules developed earlier ${ }^{8}$ for the real equation and have formulated a set of conjectures which can be tested against numerical solutions of Eq. (1). The basic idea is that the selected front $v^{\dagger}, \omega^{\dagger}$ discussed above is the entity which controls the behavior of the system. 
Moreover, we conjecture that the selected front is precisely the one obtained from the Ansatz (5), so that we can calculate $v^{\dagger}$ analytically for given parameter values. Whenever a solution of (5) is found with $v^{\dagger}>0$, a localized initial condition will lead to a positive front $(N$ invades $L$ ) given by that solution, and pulses will be unstable. If the $N$ state thus created is Benjamin-Feir unstable $^{13}$ (as can be ascertained analytically ${ }^{13}$ from the values of $\left.a_{N}^{+}, q_{N}^{+}\right)$, the front will not translate uniformly but its time-averaged velocity is expected to be close to $v^{\dagger}$. When $v^{\dagger}<0$, on the other hand, or when no solution of (5) exists, the outcome is somewhat more dependent on initial conditions, but there is usually a significant parameter range where stable stationary pulses are found. Alternatively, a localized initial condition (even of large amplitude) might decay to zero, or lead to a chaotic pulse.

Figure 1 illustrates the different regimes for fixed $c_{i}$ as a function of $\epsilon$. For $\epsilon>0$ and $c_{i} \neq 0$ [Figs. 1(c) and $1(\mathrm{~d})]$ the selected-front velocity is $\max \left(v^{*}, v^{\dagger}\right)$, where $v^{*}$ is given by the linear-marginal-stability criterion ${ }^{8,14}$ and $v^{+}$is obtained from (5). Note that the transition point $\epsilon=\epsilon^{\dagger}$ where $v^{*}=v^{\dagger}$, which was found ${ }^{8}$ to be $\epsilon^{\dagger}=0.75$ in the real case, now depends on the coefficients $c_{i}$. For $\epsilon<0$ the point $\epsilon=\epsilon_{1}=-\frac{3}{16}$, which marks the transition between the selected front $v^{\dagger}$ and the $A=0$ solution in the real case [Fig. 1(a)], now opens up into a finite region $\epsilon_{2}<\epsilon<\epsilon_{3}$, in which stable pulses exist [Fig. 1(c)]. Although both $\epsilon_{2}$ and $\epsilon_{3}$ depend on the $c_{i}$, it is only $\epsilon_{3}$ that we can calculate analytically, from the condition $v^{\dagger}\left(\epsilon_{3}\right)=0$ [see Fig. 1(d)]. Interestingly, for a large range of values of the $c_{i}$, we find ${ }^{6} \epsilon_{3}=0$, in which case positive fronts do not propagate at all below threshold, in contrast to the real equation.

We have tested this surprisingly simple picture by direct numerical integration of Eq. (1). The solid circles in Figs. 1(c) and 1(d) show the wave vector $q_{N}^{+}$and velocity $v^{+}$of pulses found for a particular choice of $c_{i}$ by starting from localized initial conditions with large enough amplitudes in the range $\epsilon_{3}<\epsilon<\epsilon^{\dagger}$, in excellent agreement with the prediction of the Ansatz (5). The front velocity obtained for $\epsilon>\epsilon^{\dagger}$ is shown by the solid triangles and follows from the linear-marginal-stability criterion. ${ }^{8,14}$ In the range $\epsilon_{2}<\epsilon<\epsilon_{3}$ stable pulses were obtained, as indicated by the crosses. The exact pulse solution (6) can be shown ${ }^{6}$ to exist only for $\epsilon>\epsilon_{3}$, so it is always unstable to front generation.

Another way to present our results is to fix $\epsilon$ and plot the dependence of velocity on the $c_{i}$, e.g., the variation with $c_{3}$ at fixed $c_{1}$ and $c_{5}$ as in Fig. 2. For $\epsilon<0$ [Fig. 2(a)], the Ansatz (5) leads to a velocity $v^{+}$(solid line) which exists over a finite band of $c_{3}$, corresponding to the band of existence of the wave vector $-\left(\frac{1}{4}+\epsilon\right)^{1 / 2}<q_{N}^{\dagger}$ $<\left(\frac{1}{4}+\epsilon\right)^{1 / 2}$. A numerical simulation of Eq. (1) in the range $c_{3}<c_{3}^{(0)}=1.22$, where $v^{+}>0$, yields the solid circles which are again in excellent agreement with the an-

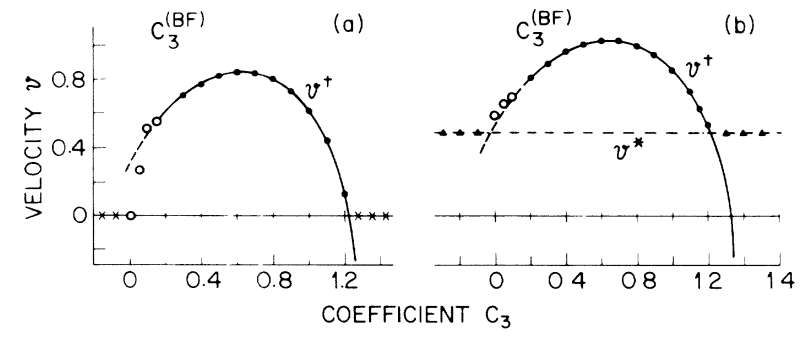

FIG. 2. Front velocity vs parameter $c_{3}$ of the GL equation, for fixed $c_{1}=1, c_{5}=-0.8$, and (a) $\epsilon=-0.03$, (b) $\epsilon=+0.03$. The velocity $v^{+}$obtained from the selected-front Ansatz (5) is shown by the solid line, and the linear-marginal-stability velocity $v^{*}$ is given by the dot-dashed line. For $c_{3}<c_{3}^{(B F)}$ the nonlinear state $a_{N}^{\dagger}, q_{N}^{\dagger}$ produced by (5) is Benjamin-Feir unstable. The symbols represent numerical solutions of (1) and have the same meaning as in Fig. 1. The open circles are timeaveraged velocities of chaotic solutions.

alytic prediction. For $c_{3}>c_{3}^{(0)}$, where $v^{\dagger}<0$ ( $L$ invades $N)$, we find that stationary pulses are stable as indicated by the crosses, whereas they are unstable for $c_{3}<c_{3}^{(0)}$. For $\epsilon>0$, Fig. 2(b), the numerical calculation yields a positive front propagating with a velocity given by $\max \left(v^{*}, v^{\dagger}\right)$, as indicated by the circles and triangles. At the edges of the band of existence of $v^{+}$, e.g., for $c_{3}<c_{3}^{(\mathrm{BF})}$ in Fig. 2, the nonlinear state $a_{N}^{+}, q_{N}^{+}$is Benjamin-Feir (BF) unstable ${ }^{13}$ and the moving-front solution found numerically is also not strictly periodic, the more so as $c_{3}$ goes further below $c_{3}{ }^{(\mathrm{BF})}$. The timeaveraged velocity, nevertheless, initially follows $v^{\dagger}$ rather closely, as indicated by the open circles, even through the fluctuations about the average velocity can be large.

Our predictions are also in accord with numerical work of previous authors: (i) The parameter values chosen by Thual and Fauve ${ }^{5}$ correspond to $c_{1}=0, c_{3}$ $=0.333, \quad c_{5}=0.364$, and $-0.138 \leq \epsilon \leq-0.031$, for which the Ansatz (5) has no solution. For slightly smaller values of $c_{1}$, however, solutions with $v^{\dagger}<0$ do appear, so it is not surprising that stable pulses were found by these authors. (ii) The calculation in Fig. 8 of Deissler ${ }^{3}$ corresponds to $c_{1}=-2.5, c_{3}=-0.5, c_{5}=-2$, and $\epsilon$ $=-0.125$, for which (5) also has no solution. Moreover, the solution is relatively far away in parameter space, and all nonlinear states $a_{N}, q_{N}$ are BF unstable. ${ }^{13}$ In this case Deissler found a chaotic pulse in his simulation, ${ }^{3}$ though we have also obtained stable stationary pulses for these parameters, starting from more localized initial conditions. ${ }^{6}$

Quantitative applications of our results to binary-fluid convection or plane Poiseuille flow must await a more detailed determination of the validity of the GL equation. At this stage it is already clear, however, that our work is unlikely to explain directly the experimental observations by Kolodner, Bensimon, and Surko ${ }^{1}$ of stationary front pairs in binary-fluid convection. These 
fronts were found to have arbitrary separation for a range of $\epsilon$ values. In the GL equation (1), a pair of stationary fronts can be obtained ${ }^{15}$ from a stationary pulse by requiring the width of the pulse to diverge, but when this condition is approximately satisfied the distance between the two fronts will be a fixed function of the parameters $\left\{\epsilon, c_{l}\right\}$, in seeming contradiction to the experimental findings. ' Another aspect of our work which limits its direct applicability to binary-fluid convection, even to explain the observation ${ }^{16}$ of stationary pulses, is our consideration of only one direction of propagation of waves in (1), and the consequent neglect of the groupvelocity term $s \partial_{x} A$ (if right- and left-propagating waves are present this term cannot be transformed away). In our model the stationary pulses are at rest in the frame moving with velocity $s$; whether the more general model will have pulses at rest in the laboratory frame remains to be seen. For plane Poiseuille flow the observed existence of turbulent bursts is qualitatively consistent with predictions based on Eq. (1), as noted by Deissler. ${ }^{3}$ However, his estimates ${ }^{17}$ of convective versus absolute instability near onset, which are based on $v^{*}$, may have to be revised since fronts may propagate faster than $v^{*}$ over an appreciable parameter range.

In conclusion, we have presented some conjectures concerning the behavior of pulses and fronts in the complex GL equation (1), and verified their predictions by direct simulation. An important question which remains to be elucidated is whether the simple Ansatz (5) produces the selected front $v^{\dagger}$ in all cases, and why it succeeds or fails when it does. It seems likely that the perturbative methods ${ }^{18}$ discussed by Fauve and Thual ${ }^{19}$ and by Hakim, Jakobsen, and Pomeau, ${ }^{15}$ could shed light on these questions.

The authors thank Y. Pomeau and S. Fauve for communicating their work prior to publication. P.C.H. acknowledges the hospitality of the Aspen Center for Physics.

Note added.-The recent results of Brand and Deissler ${ }^{20}$ on pulse interactions in two coupled GL equations have a natural explanation in terms of our work. When the two pulses do not overlap the system studied by these authors is equivalent to two uncoupled versions of Eq. (1), which admit discrete pulse solutions moving with the group velocity (the equivalent of our stationary pulses). It is thus to be expected that after a collision the pulses will either emerge unchanged, or disappear, or form another discrete solution. We are indebted to $M$. C. Cross for bringing this point to our attention.

${ }^{1}$ P. Kolodner, D. Bensimon, and C. M. Surko, Phys. Rev.
Lett. 60, 1723 (1988); J. Fluid Mech. (to be published); E. Moses, J. Fineberg, and V. Steinberg, Phys. Rev. A 35, 2757 (1987); R. Heinrichs, G. Ahlers, and D. S. Cannell, Phys. Rev. A 35, 2761 (1987).

${ }^{2}$ See M. J. Landman, Stud. Appl. Math. 76, 187 (1987), and references therein.

${ }^{3}$ R. J. Deissler, J. Stat. Phys. 54, 1459 (1989).

${ }^{4}$ J. J. Hegseth, C. D. Andereck, F. Hayot, and Y. Pomeau, Phys. Rev. Lett. 62, 257 (1989).

${ }^{5}$ O. Thual and S. Fauve, J. Phys. (Paris) 49, 1829 (1988).

${ }^{6} \mathrm{~W}$. van Saarloos and P. C. Hohenberg (to be published).

${ }^{7}$ By "complex" and "real" we refer to the coefficients in the equation. In both cases the order parameter itself is complex.

${ }^{8}$ See W. van Saarloos, Phys. Rev. A 37, 211 (1988); 39, 6367 (1989), and references therein.

${ }^{9}$ The nomenclature for these structures is not uniform in the literature: pulses are also referred to as solitons or $s$ waves, fronts are known as kinks or shocks, and domain walls are called shocks, sources, sinks, or holes.

${ }^{10}$ The results stated here hold over most of the parameter range. For large positive $\epsilon$ there is a richer multiplicity of solutions which will be discussed in Ref. 6 .

${ }^{11}$ Note that an exact solution of the form (5) still exists if a term proportional to $|A|^{2} \partial_{x} A$ is added to (1).

${ }^{12}$ L. M. Hocking and K. Stewartson, Proc. Roy. Soc. London A 326, 289 (1972).

${ }^{13}$ J. T. Stuart and R. C. DiPrima, Proc. Roy. Soc. London A 362, 27 (1978).

${ }^{14}$ It should be noted that for the complex equation the front obtained by linear marginal stability does not have the form (2) of a uniformly translating solution.

${ }^{15}$ As noted by V. Hakim, P. Jakobsen, and Y. Pomeau [Europhys. Lett. (to be published)], one can define two classes of stationary pulse solutions, one stable and the other one unstable. A stable pulse of infinite width is obtained by joining up two selected fronts placed symmetrically about $x=0$, and requiring that these fronts be stationary $\left(v^{+}=0\right)$. This condition defines a codimension-one subspace of the parameter set $\left\{\epsilon, c_{l}\right\}$, as found by Hakim, Jakobsen, and Pomeau. Note, however, that the stationary selected fronts will, in general, have nonzero wave vectors $q^{\dagger}\left(v^{\dagger}\right)$, and the wide pulse will have a domain boundary (or shock) at $x=0$ formed by joining the $q^{\dagger}$ and $-q^{\dagger}$ solutions. A similar structure has been seen in the experiments of Kolodner, Bensimon, and Surko (Ref. 1), but a continuum of stationary front pairs without a shock in the center was also frequently observed. For our model the condition that the two fronts join smoothly $\left[q^{\dagger}\left(v^{\dagger}=0\right)=0\right]$ defines a codimension-two subspace, which makes the experimental findings even more surprising.

${ }^{16}$ J. J. Niemela, G. Ahlers, and D. S. Cannell (unpublished).

${ }^{17}$ R. J. Deissler, Phys. Fluids 30, 2303 (1987).

${ }^{18}$ A. C. Newell, Rocky Mountain J. Math. 8, 25 (1978); Y. S. Kivshar and B. A. Malomed, Rev. Mod. Phys. 61, 763 (1989).

${ }^{19} \mathrm{~S}$. Fauve and O. Thual, Phys. Rev. Lett. 64, 282 (1990).

${ }^{20} \mathrm{H}$. Brand and R. J. Deissler, Phys. Rev. Lett. 63, 2801 (1989). 